\title{
Elaboración de biocombustibles sólidos densificados a partir de la mezcla de dos biomasas residuales, un aglomerante a base de yuca y carbón mineral, propios del departamento de Córdoba
}

\author{
Development of densified solid biofuels from mixture of two residual biomass, \\ a cassava starch and coal, own of department of Córdoba \\ Camilo Andrés Hoyos Álvarez ${ }^{1} \quad$ Yahir Enrique González Doria ${ }^{1}$ \\ Jorge Mario Mendoza Fandiño ${ }^{1 *}$ \\ Recibido 5 de septiembre de 2017, aceptado 11 de junio de 2018 \\ Received: September 5, 2017 Accepted: June 11, 2018
}

\begin{abstract}
RESUMEN
En este trabajo se elaboraron biocombustibles sólidos densificados a partir de la mezcla de cascarilla de arroz, fibra de coco, carbón mineral y un aglomerante a base de yuca, propios del departamento de Córdoba. Inicialmente, se elaboró un diseño experimental simplex reticular ampliado, para determinar el número de corridas. Luego, se realizó el proceso de densificación manual, las mezclas fueron compactadas con una presión de 6000 Psi (41,36 MPa) durante un tiempo de sostenimiento de la carga de 15 minutos. Posteriormente se realizaron pruebas de durabilidad mecánica y resistencia a la compresión a las briquetas fabricadas, y se calculó analíticamente el poder calorífico y las emisiones de azufre de cada corrida. Se encontró que el carbón es el componente que más afecta negativamente la durabilidad de las briquetas, contrario a la fibra de coco, la cual contribuye a mejorar dicha propiedad. Todas las briquetas presentaron excelente resistencia a la compresión, además ninguna superó en $1 \%$ las emisiones de azufre.
\end{abstract}

Palabras clave: Biocombustibles, aglomerante, durabilidad, poder calorífico, briquetas.

\section{ABSTRACT}

In this paper densified solid biofuels were produced from the mixture of rice husk, coir, coal and a cassava binder, own department of Cordoba. Initially, it was developed an expanded lattice simplex experimental design to determine the number of runs. Manual densification process was performed; the mixtures were compacted with a pressure of $6000 \mathrm{psi}(41,36 \mathrm{MPa})$ for a hold time of $15 \mathrm{~min}$ load. Later tests of mechanical and compressive strength to the briquettes manufactured durability were performed, and analytically calculated the heating value and sulfur emissions each run.

It was found that coal is the component that most adversely affects the durability of briquettes, contrary to coconut fiber, which helps to improve the property. All briquettes showed excellent compressive strength, besides no sulfur emissions exceeded $1 \%$.

Keywords: Biofuel, binder, durability, heat value, briquettes.

1 Departamento Ingeniería Mecánica. Universidad de Córdoba. Carrera 6 \# 76-103. Montería, Colombia.

E-mail: caanhoal@hotmail.com; yahirgonzalez@correo.unicordoba.edu.co; jorge.mendoza@correo.unicordoba.edu.co 


\section{INTRODUCCIÓN}

El departamento de Córdoba es reconocido por la fertilidad de sus suelos y su gran variedad de cultivos, entre los cuales se encuentran el arroz y el coco. La producción anual de coco en la región es de 59164 toneladas, cultivados en un área total de 3788 hectáreas mientras que 8158 hectáreas son utilizadas en la siembra de arroz con una producción anual aproximada de 17751 toneladas [14, 2].

Estos cultivos generan grandes cantidades de residuos sólidos que se convierten en un problema para los productores, más que todo por los altos volúmenes que dichos residuos representan, dada su baja densidad y además no se obtiene ningún valor agregado de ellos, lo cual implica sobrecostos de manejo y transporte. Esto conlleva a que los agricultores tomen la decisión de quemarlos a cielo abierto o arrojarlos a fuentes hídricas, ocasionando así gran impacto ambiental en cualquiera de los casos.

También se sabe que el departamento de Córdoba cuenta con una mina de carbón mineral llamada La Guacamaya, de la cual se extraen aproximadamente 400000 ton/año. Este carbón se caracteriza por tener un contenido aproximado de azufre de 1,3\% lo cual impide que se utilice como combustible en estado puro según la resolución 623 de 1998 del ministerio de ambiente colombiano, que establece que dicho porcentaje no debe exceder de $1 \%$ [6].

Es por esto que existe la necesidad de buscar un método de aprovechamiento del potencial energético del carbón y la biomasa producida en la región, conociendo que la cascarilla de arroz, la fibra de coco y el carbón tienen un poder calorífico de 15800 , 16300 y $23900 \mathrm{KJ} / \mathrm{Kg}$ respectivamente, lo cual les brinda un atractivo como fuente de energía renovable.

En los últimos años han surgido diversas tecnologías en la búsqueda de nuevas fuentes de energía para suplir la creciente demanda y que al mismo tiempo sean más amigables con el medio ambiente. Según Liu y otros autores, una de éstas alternativas es el aprovechamiento del potencial energético presente en las biomasas residuales [13]. Trabajos como el de M. Stolarki y otros autores afirman que en diversas zonas de la Unión Europea se han utilizado cada vez más a menudo fuentes renovables en la generación de energía [23]. Adicionalmente, otras investigaciones han evidenciado el potencial de la biomasa residual de productos agrícolas como fuente renovable de energía. Grover y otros autores, asi como Demirbas y otros autores demostraron que los residuos agrícolas como la cascarilla de arroz o la tusa de maíz pueden ser densificados para construir briquetas combustibles y aprovechar su poder calorífico [7, 3]. Francis menciona también que ésta biomasa puede contribuir a la hora de suplir la demanda de energía en las zonas rurales, al usarse como combustibles densificados en forma de briquetas [5]. Muazu y otros autores estudiaron la durabilidad de briquetas hechas de la mezcla de cascarilla de arroz y tusa de maíz, y un agente aglomerante a base de almidón de yuca [18]. Realizaron un proceso de caracterización para determinar la densidad, contenido de humedad, tamaño de partículas, absorción de agua y gravedad específica de las biomasas. Luego, llevaron a cabo un proceso de densificación por medio de una prensa hidráulica en una matriz cilíndrica. Pudieron confirmar que el alto contenido de tusa de maíz y las altas presiones de compactación, inciden de manera positiva en la resistencia a la compresión y la durabilidad de las briquetas elaboradas bajo estos parámetros. [8] K. Ishii y otros determinaron la influenciadel contenido de humedad, tamaño de partícula y temperatura para la producción de pellets realizando experimentos con paja de arroz. Concluyendo que las condiciones óptimas fueron humedad de $13 \%-15 \%$, una temperatura de $60-80{ }^{\circ} \mathrm{C}$ y un tamaño de partícula que va entre 10 y 20 $\mathrm{mm}$. [8]. Otras investigaciones $20 \mathrm{~mm}$. [8]. Otras investigaciones han determinado que algunos de los factores que pueden influenciar en la calidad de la densificación de pellets y briquetas son la presión de compactación, el contenido de humedad y el tamaño de partícula. [9-11, 22]. Así mismo, Molina $\mathrm{y}$ otros autores fabricaron briquetas con residuos de muebles compuestos de madera, espuma y tapicería, aprovechando su alto valor calorífico. Teniendo en cuenta variables como contenido de humedad y presión de compactación, se concluyó que la presión de compactación y porcentaje de humedad óptimo fueron de $44 \mathrm{MPa}$ y $8 \%$ respectivamente [17].

Li y otros autores afirman que la presencia de humedad en la biomasa es necesaria, ya que actúa como un agente de unión. Sin embargo, cuando el contenido de humedad es alto, los materiales densificados tienen una durabilidad menor y 
fácilmente se fracturan, Determinaron que el rango de humedad para producir buena calidad del material densificado (madera dura, madera blanda y corteza en forma de aserrín) es de 5-12\% para todos los materiales leñosos estudiados; el contenido óptimo de humedad fue de alrededor de $8 \%$ [12]. Ortega y otros autores realizaron procesos de densificación a partir de la mezcla de cascarilla de arroz, carbón mineral y aglomerante natural de yuca, usando un tamaño de partícula de $1,18 \mathrm{~mm}$ y una presión de compactación de $7 \mathrm{MPa}$ durante un tiempo de sostenimiento de la carga de 10 min. Encontraron que no fue posible desarrollar briquetas durables y resistentes a partir de esta mezcla [20].

Con la ejecución de este proyecto se busca elaborar biocombustibles sólidos densificados (briquetas) a partir de la mezcla de cuatro componentes: biomasas residuales de los cultivos de coco y arroz, carbón mineral y un agente aglomerante a base de yuca, con el objetivo de que estas alcancen parámetros de durabilidad mecánica y resistencia a la compresión aceptados por las normas CEN/TS 15210 y NTC 2060, respectivamente, que les permitan resistir condiciones de transporte y manipulación. Adicionalmente, con la presencia de carbón en la mezcla, se pretende que las briquetas adquieran un poder calorífico superior al de la cascarilla de arroz en estado puro, sin perder de vista que durante la combustión deben presentarse emisiones de azufre en niveles inferiores al $1 \%$.

\section{MATERIALES Y MÉTODOS}

Análisis próximo y elemental: Para conocer con precisión las propiedades y composición químicas de la materia prima utilizada en la elaboración de las briquetas, se tomaron muestras de cada biomasa y de carbón mineral y se realizaron dos tipos de análisis: el primero, un análisis elemental, con el fin de conocer el contenido de carbono, hidrógeno, nitrógeno y azufre presentes en los dos tipos de biomasa y el carbón mineral. Seguidamente, se realizó un análisis próximo, para determinar el porcentaje de humedad residual, cenizas, material volátil, carbono fijo, azufre total y el poder calorífico superior de una muestra de cada biomasa y una de carbón. Estos análisis se realizan para delimitar el experimento y garantizar así que los resultados obtenidos se cumplen para las mezclas de carbón y biomasa que tengan las propiedades fisicoquímicas que presentaron las muestras sometidas a evaluación.
Molienda y tamizado: Para la elaboración de las briquetas, lo primero que se hizo fue preparar la biomasa y el carbón realizando un proceso de molienda con un molino de martillos, el cual tiene una potencia nominal de $10 \mathrm{HP}$, para así llevarlo a un tamaño de partícula menor a $3 \mathrm{~mm}$ recomendado por Fonseca y otros autores, con el fin de garantizar la compactación y el acabado superficial [4].

Luego de esto se realizó un proceso de tamizado siguiendo la norma ASTM-E11 para obtener partículas más finas. Se trabajó con los siguientes tamaños:

- $625 \mu \mathrm{m}$ para la cascarilla de arroz y el carbón.

- 450 um para la fibra de coco.

Diseño de experimento: Esto se hizo con el fin de determinar el número de corridas y las proporciones en masa de cada factor en cada una de las mezclas, para esto se estableció el porcentaje de biomasa, porcentaje de aglomerante y porcentaje de carbón como los factores y la durabilidad, resistencia a la compresión, poder calorífico y porcentaje de azufre como las variables de respuesta. Se escogió un modelo simplex reticular debido a que este es un diseño experimental típico para mezclas de cuatro componentes, por otra parte, se definieron los límites de los factores los cuales se muestran en la Tabla 1.

Tabla 1. Rangos de trabajo para el contenido de cada factor.

\begin{tabular}{|l|c|}
\hline \multicolumn{1}{|c|}{ Factor } & $\begin{array}{c}\text { Rango de Trabajo } \\
(\boldsymbol{\%})\end{array}$ \\
\hline Fibra de coco & $40-70$ \\
\hline Cascarilla de arroz & $0-20$ \\
\hline Carbón mineral & $0-25$ \\
\hline Aglomerante a base de yuca & $5-15$ \\
\hline
\end{tabular}

Fuente: Autores (2016).

Varias fueron las consideraciones tenidas en cuenta al momento de elegir este agente aglomerante, entre las que se encuentran: la amplia disponibilidad comercial, el bajo costo de producción, la fácil obtención de la materia prima dado que es un cultivo propio del departamento de Córdoba, es considerado un buen agente de unión debido a sus propiedades químicas y estructurales [21] y ha arrojado buenos resultados en investigaciones previas en las cuales ha sido utilizado 
para la fabricación de briquetas a base de otras biomasas como residuos de cosecha de sorgo y tusa de maíz.

El contenido de aglomerante se definió con base en los resultados previamente obtenidos en diversas investigaciones en las que se utilizó el mismo tipo de sustancia, como la Mauzu donde el aglomerante se utilizó en 10\%; o la de Ortega y Berrastegui donde el rango de trabajo fue de 5-20\% [18, 20].

Elaboración de briquetas: El proceso de briquetado se realizó a una temperatura ambiente promedio de $32^{\circ} \mathrm{C}$, elaborando 4 réplicas por cada mezcla del diseño experimental, de las cuales 2 se utilizaron para prueba de resistencia a la compresión y 2 para prueba de durabilidad. Cada briqueta se hizo considerando una masa de $50 \mathrm{~g}$ y un diámetro fijo en la máquina de briquetado de $52 \mathrm{~mm}$. La presión de compactación fue de $14 \mathrm{MPa}$, con un tiempo de sostenimiento de la carga de 15 minutos, la briqueta fue retirada del equipo después de 3 minutos, con el fin de contrarrestar los efectos de expansión volumétrica tras liberar a la briqueta de la presión aplicada (ver Figura 1).

Pruebas mecánicas: Luego de la elaboración de todas las briquetas, estas fueron sometidas a pruebas de resistencia a la compresión y durabilidad mecánica, con el fin de medir la capacidad de estas a permanecer intactas frente a procesos de manipulación y transporte. El ensayo de resistencia a la compresión se realizó utilizando una máquina para ensayos a compresión marca PINZUAR (ver Figura 2) con capacidad de $1200 \mathrm{KN}$ y resolución de $0,01 \mathrm{KN}$, aplicando la carga hasta la falla de la briqueta. Los resultados en la prueba, se definieron a partir del requerimiento de resistencia al aplastamiento que debe tener una briqueta combustible, establecido

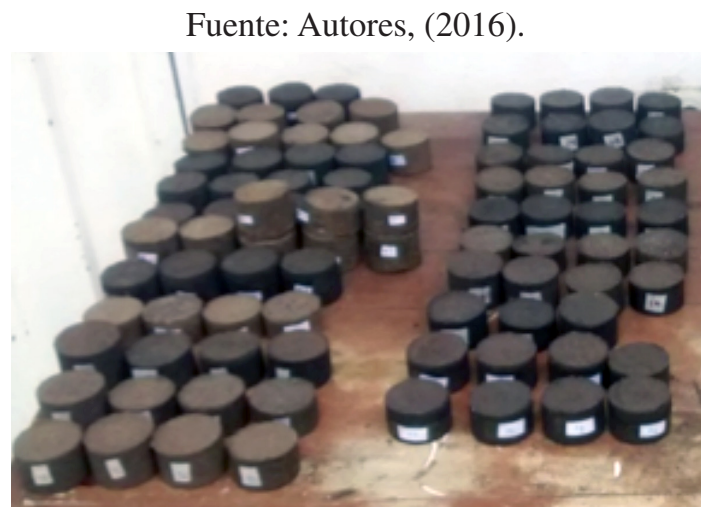

Figura 1. Briquetas terminadas.

en el numeral 4.2.1 de la norma técnica colombiana NTC-2060.

Por otra parte, la prueba de Durabilidad se desarrolló bajo la Norma CEN/TS 15210, en la cual las briquetas se sometieron a golpes controlados entre sí y contra las paredes de una cámara rotativa que gira a una velocidad de 21 RPM durante 5 minutos (Ver Figura 3).

Cálculo de poder calorífico y emisiones de azufre de las briquetas: Ambos cálculos se hicieron de forma analítica, dado que para combustibles líquidos y sólidos es muy frecuente utilizar ecuaciones empíricas o ecuaciones que obtienen el poder calorífico inferior o superior en función de las fracciones másicas de los componentes elementales [15-16].

Para el poder calorífico de las briquetas se tuvo en cuenta el porcentaje en masa de cada factor y el poder calorífico de cada uno de los factores. Para el cálculo de las emisiones de azufre también se tuvo en cuenta el porcentaje en masa de cada componente y las emisiones

Fuente: Autores (2016).
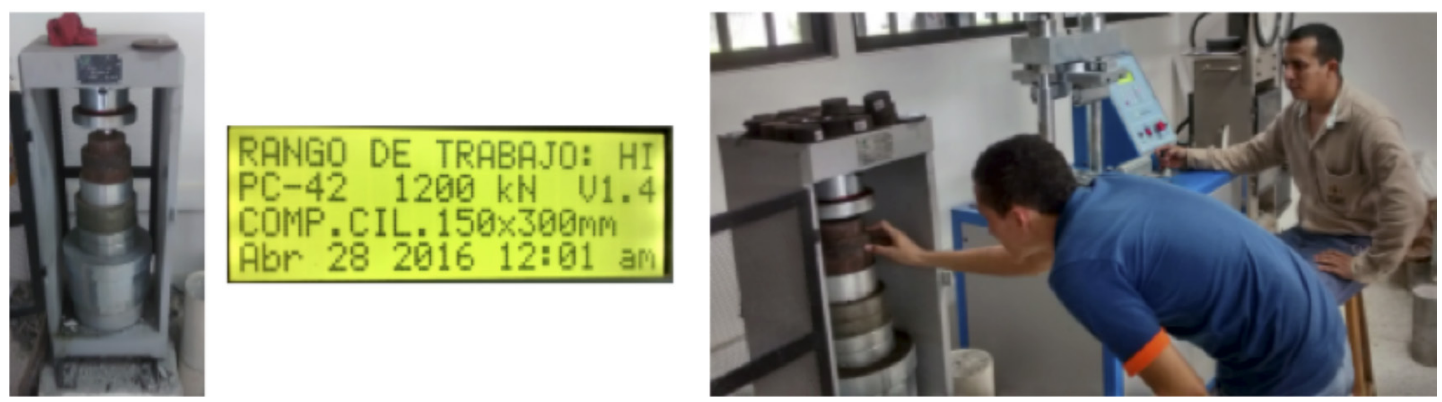

Figura 2. Máquina para ensayos a compresión PINZUAR. 
Fuente: Autores (2016).

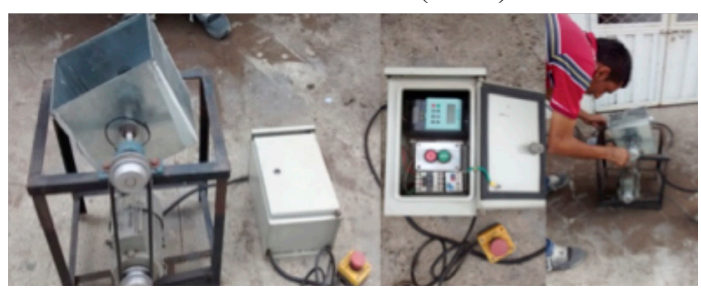

Figura 3. Máquina para ensayos de durabilidad mecánica.

de azufre de cada factor. Tanto el poder calorífico como las emisiones de azufre de cada componente se obtuvieron a partir de los análisis próximo y elemental como se nota en las ecuaciones (1) y (2).

$$
\begin{gathered}
P C=(16,3117 * \% F C)+(23,9275 * \% C) \\
(15,8135 * \% C A)+(10,3413 * \% A) \\
\% S=(0,12 * \% F C)+(1,55 * \% \mathrm{C}) \\
(0,10 * \% C A)+(0 * \% A)
\end{gathered}
$$

Donde:

$\mathbf{P C}=$ poder calorífico.

$\% \mathbf{S}=$ porcentaje de emisiones de azufre.

$\% \mathbf{F C}=$ porcentaje en masa de la fibra de coco.

$\% \mathbf{C}=$ porcentaje en masa del carbón.

$\% \mathbf{C A}=$ porcentaje en masa de la cascarilla de arroz .

$\% \mathbf{A}=$ porcentaje en masa del aglomerante.

Determinación de las mezclas favorables: Luego de cumplir con toda la fase experimental, los datos obtenidos para cada variable de salida fueron consignados y procesados en el software de análisis estadístico Desing Expert versión 10, usando para ello un modelo simplex reticular, debido a su capacidad para determinar el comportamiento de un diseño de experimento para mezclas con dos o más factores. El software generó las respectivas coordenadas triangulares para la durabilidad y resistencia a la compresión y posteriormente se ejecutó una optimización multivariable, maximizando y minimizando ciertos factores y ciertas variables de salida, con base en ciertos parámetros como se muestra en la Tabla 2.

Posteriormente, se tomó una muestra de la mezcla óptima seleccionada y se le realizaron pruebas de azufre total bajo la norma ASTM D4239-14 método A; y de poder calorífico según la norma ASTM D5865-13.

\section{RESULTADOS Y DISCUSIONES}

Análisis próximo y elemental: Según lo muestra la Tabla 3, el mayor poder calorífico lo presenta la muestra de carbón, como era de esperarse, al igual que el mayor contenido de azufre. También es de notar el elevado poder calorífico que presenta la muestra de fibra de coco, lo cual la hace una biomasa con un atractivo potencial energético. El poder calorífico de la cascarilla de arroz, aunque menor, es también elevado y muy cercano al de la fibra de coco, y a su vez se evidencia que el contenido de azufre de esta muestra es el menor de todos, lo cual la hace una opción tentativa a la hora usarla como una fuente de generación alternativa de energía por medio de procesos de combustión.

Batería de ensayo: En la Tabla 4, se presenta la batería de ensayo arrojada por el software de acuerdo a los límites establecidos en el porcentaje de los factores, además se muestran los resultados

Tabla 2. Parámetros de optimización.

\begin{tabular}{|l|c|c|c|}
\hline \multicolumn{1}{|c|}{ Nombre } & Objetivo & Límite inferior & Límite superior \\
\hline Fibra de coco (\%) & Maximizar & 40 & 70 \\
\hline Carbón (\%) & Maximizar & 0 & 25 \\
\hline Cascarilla de arroz $(\%)$ & Maximizar & 0 & 20 \\
\hline Aglomerante (\%) & En el rango & 5 & 15 \\
\hline Carga (KN) & Maximizar & 4,62 & 17,19 \\
\hline Durabilidad (\%) & Maximizar & 90 & 100 \\
\hline Poder calorífico (MJ/Kg) & Maximizar & 15,3 & 17,9 \\
\hline Emisiones de azufre (\%) & Minimizar & 0,098 & 1 \\
\hline
\end{tabular}

Fuente: Design Expert versión 10. 
Tabla 3. Resultados de los análisis próximo y elemental para las materias primas.

\begin{tabular}{|l|c|c|c|c|c|c|c|c|c|}
\hline \multicolumn{1}{|c|}{ Muestra } & $\begin{array}{c}\text { Humedad } \\
\text { residual (\%) }\end{array}$ & $\begin{array}{c}\text { Cenizas } \\
(\%)\end{array}$ & $\begin{array}{c}\text { Materia } \\
\text { volátil (\%) }\end{array}$ & $\begin{array}{c}\text { Carbono } \\
\text { fijo (\%) }\end{array}$ & $\begin{array}{c}\text { Azufre } \\
\text { total (\%) }\end{array}$ & $\begin{array}{c}\text { Poder calorífico } \\
\text { superior (cal/g) }\end{array}$ & $\mathbf{\% C}$ & $\mathbf{\% H}$ & $\mathbf{\% N}$ \\
\hline Fibra de coco & 13,94 & 5,10 & 57,24 & 23,72 & 0,12 & 3896 & 39,40 & 4,27 & 0,70 \\
\hline $\begin{array}{l}\text { Cascarilla de } \\
\text { arroz }\end{array}$ & 10,18 & 8,33 & 64,67 & 16,82 & 0,10 & 3777 & 39,27 & 4,91 & 0,59 \\
\hline Carbón mineral & 12,52 & 3,96 & 43,54 & 39,98 & 1,55 & 5715 & 60,29 & 4,61 & 1,47 \\
\hline
\end{tabular}

Fuente: Autores (2016).

Tabla 4. Batería de ensayos y resumen de las variables de respuesta.

\begin{tabular}{|c|c|c|c|c|c|c|c|c|}
\hline Corrida & $\begin{array}{l}\text { Fibra de coco } \\
\qquad(\%)\end{array}$ & $\begin{array}{c}\text { Carbón } \\
(\%)\end{array}$ & $\begin{array}{c}\text { Arroz } \\
(\%)\end{array}$ & $\begin{array}{l}\text { Agl. } \\
(\%)\end{array}$ & $\begin{array}{c}\text { Emisiones } \\
(\%)\end{array}$ & $\begin{array}{c}\text { P.C. } \\
(\mathrm{Mj} / \mathrm{Kg})\end{array}$ & $\begin{array}{c}\text { Durabilidad } \\
(\%)\end{array}$ & $\begin{array}{r}\text { Carge } \\
(\mathbf{K N})\end{array}$ \\
\hline 1 & 70,0 & 25,0 & 0,0 & 5,0 & 0,47 & 17,91 & 10,1 & 5,78 \\
\hline 2 & 70,0 & 5,0 & 20,0 & 5,0 & 0,18 & 16,28 & 64,5 & 9,4 \\
\hline 3 & 70,0 & 0,0 & 20,0 & 10,0 & 0,10 & 15,61 & 94,5 & 14,45 \\
\hline 4 & 50,0 & 25,0 & 20,0 & 5,0 & 0,47 & 17,81 & 35,1 & 6,78 \\
\hline 5 & 40,0 & 25,0 & 20,0 & 15,0 & 0,46 & 17,21 & 15,6 & 7,83 \\
\hline 6 & 70,0 & 15,0 & 0,0 & 15,0 & 0,32 & 16,55 & 93,1 & 10,78 \\
\hline 7 & 70,0 & 0,0 & 15,0 & 15,0 & 0,10 & 15,33 & 39,6 & 11,3 \\
\hline 8 & 60,0 & 25,0 & 0,0 & 15,0 & 0,46 & 17,31 & 65,7 & 7,95 \\
\hline 9 & 65,0 & 0,0 & 20,0 & 15,0 & 0,10 & 15,31 & 95,7 & 16,37 \\
\hline 10 & 66,4 & 19,2 & 6,4 & 8,1 & 0,38 & 17,25 & 89,5 & 4,62 \\
\hline 11 & 66,4 & 9,2 & 16,4 & 8,1 & 0,24 & 16,44 & 90,6 & 16,38 \\
\hline 12 & 66,4 & 6,7 & 16,4 & 10,6 & 0,20 & 16,10 & 92,6 & 15,82 \\
\hline 13 & 56,4 & 19,2 & 16,4 & 8,1 & 0,38 & 17,20 & 47,5 & 10,12 \\
\hline 14 & 51,4 & 19,2 & 16,4 & 13,1 & 0,38 & 16,90 & 45,3 & 15,85 \\
\hline 15 & 66,4 & 14,2 & 6,4 & 13,1 & 0,31 & 16,57 & 57,3 & 15,57 \\
\hline 16 & 66,4 & 6,7 & 13,9 & 13,1 & 0,20 & 15,96 & 95,5 & 16,07 \\
\hline 17 & 61,4 & 19,2 & 6,4 & 13,1 & 0,38 & 16,95 & 13 & 15,8 \\
\hline 18 & 63,9 & 6,7 & 16,4 & 13,1 & 0,20 & 15,95 & 95,3 & 16,68 \\
\hline 19 & 70,0 & 15,0 & 10,0 & 5,0 & 0,33 & 17,09 & 60 & 8,57 \\
\hline 20 & 60,0 & 25,0 & 10,0 & 5,0 & 0,47 & 17,86 & 44,1 & 15,48 \\
\hline 21 & 70,0 & 20,0 & 0,0 & 10,0 & 0,39 & 17,23 & 23,1 & $12,8 \mathrm{c}$ \\
\hline 22 & 65,0 & 25,0 & 0,0 & 10,0 & 0,47 & 17,61 & 4,8 & 15,75 \\
\hline 23 & 70,0 & 2,5 & 20,0 & 7,5 & 0,14 & 15,94 & 68,9 & 12,89 \\
\hline 24 & 60,0 & 15,0 & 20,0 & 5,0 & 0,32 & 17,04 & 57,6 & 14,35 \\
\hline 25 & 70,0 & 0,0 & 17,5 & 12,5 & 0,10 & 15,47 & 90,9 & 16,26 \\
\hline 26 & 67,5 & 0,0 & 20,0 & 12,5 & 0,10 & 15,46 & 91,5 & 16,03 \\
\hline 27 & 45,0 & 25,0 & 20,0 & 10,0 & 0,46 & 17,51 & 46,5 & 12,14 \\
\hline 28 & 50,0 & 25,0 & 10,0 & 15,0 & 0,46 & 17,26 & 19,1 & 9,45 \\
\hline 29 & 52,5 & 12,5 & 20,0 & 15,0 & 0,28 & 16,26 & 42,5 & 13,42 \\
\hline 30 & 70,0 & 7,5 & 7,5 & 15,0 & 0,21 & 15,94 & 68,7 & 16,32 \\
\hline 31 & 65,0 & 20,0 & 0,0 & 15,0 & 0,39 & 16,93 & 44,4 & 13,5 \\
\hline 32 & 67,5 & 0,0 & 17,5 & 15,0 & 0,10 & 15,32 & 83,5 & 15,74 \\
\hline 33 & 70,0 & 9,0 & 11,0 & 10,0 & 0,23 & 16,34 & 93,3 & 16,5 \\
\hline 34 & 63,3 & 18,3 & 13,3 & 5,0 & 0,37 & 17,33 & 85,9 & 14,72 \\
\hline 35 & 55,0 & 25,0 & 10,0 & 10,0 & 0,46 & 17,56 & 31,7 & 13,87 \\
\hline 36 & 66,7 & 21,7 & 0,0 & 11,7 & 0,42 & 17,25 & 50,6 & 10,27 \\
\hline 37 & 59,0 & 11,0 & 20,0 & 10,0 & 0,26 & 16,44 & 74,4 & 17,19 \\
\hline 38 & 68,3 & 0,0 & 18,3 & 13,3 & 0,10 & 15,41 & $\begin{array}{l}94,9 \\
\end{array}$ & 15,6 \\
\hline 39 & 61,0 & 13,0 & 11,0 & 15,0 & 0,29 & 16,34 & 40,1 & 14,35 \\
\hline 40 & 62,8 & 13,3 & 12,8 & 11,1 & 0,29 & 16,59 & 45,1 & 14,76 \\
\hline
\end{tabular}

Fuente: Autores (2016). 
obtenidos para cada una de las variables de respuesta. De los resultados de la prueba de durabilidad mecánica para cada corrida se puede decir que solo 11 (correspondientes a las filas resaltadas) de los $40(27,5 \%)$ diferentes tipos de briquetas que se elaboraron obtuvieron un porcentaje de durabilidad mayor al 90\%, el cual corresponde al valor mínimo que debe tener una briqueta para ser considerada aceptable, siendo 95,7\% el valor de durabilidad más alto correspondiente a la mezcla 9. Como se muestra en la Tabla 4, las corridas 1, 5, 17 y 22 fueron las que presentaron menor durabilidad, debido a que estas contienen altos porcentajes de carbón. De acuerdo a lo comprobado experimentalmente, las mezclas que tenían un alto contenido de carbón, presentaban mayor oposición al momento de compactarlas y, por ende, resultaban briquetas de mayor altura, lo cual se traduce en un mayor espaciamiento entre las partículas, en comparación con aquellas briquetas con un menor contenido de este factor. Así pues, se puede afirmar que la causa principal de que el carbón sea un factor detractor sobre la durabilidad mecánica en las briquetas, se debe a que mientras mayor sea el espacio entre las partículas de estas, menos resistirán a los golpes aleatorios que sufren en la prueba de durabilidad y presentarán fractura, quiebre y pérdida de masa en mayor medida que aquellos especímenes fabricados con menor proporción en masa de carbón.

De los resultados de la prueba de compresión, se puede decir que el 100\% de las mezclas superó la carga mínima de 70 Kgf necesaria para ser aceptadas, tal como lo dice la norma. Es necesario resaltar que todas las corridas superaron ampliamente esta prueba, debido a que, por la elevada presión de compactación manejada en el proceso, la relación final de altura-diámetro era inferior a 2, lo cual aumenta considerablemente la carga necesaria para lograr que estas fallen.

\section{Sistema triangular de coordenadas para la dura-} bilidad: A través de la Figura 4, se puede afirmar que para obtener una durabilidad mayor al $90 \%$, es necesario que en la mezcla haya presente altas cantidades de fibra de coco y cascarilla de arroz, consiguiéndose buenos resultados con valores de estas dos biomasas comprendidos en los rangos de $60-70 \%$ y $14-20 \%$, respectivamente. También se observa que el carbón es el elemento de la mezcla que más disminuye la durabilidad, pues a medida que aumenta el contenido de carbón en
Fuente: Design Expert versión 10.

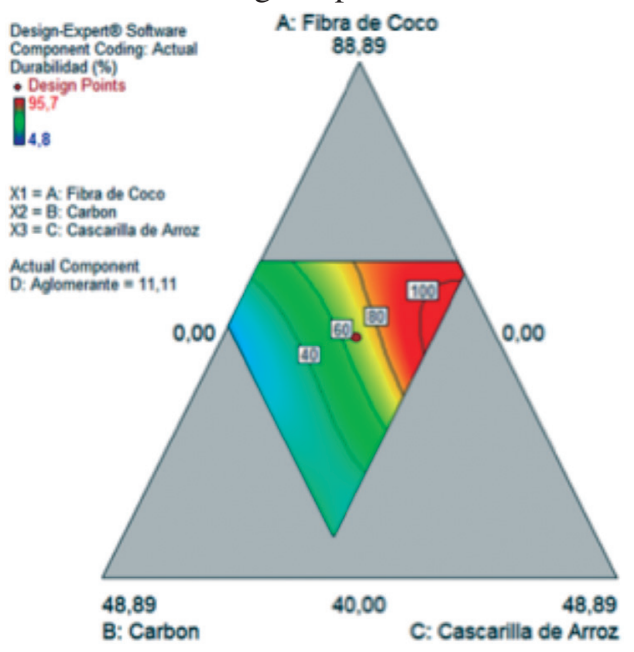

Figura 4. Gráfico de contorno para la durabilidad mecánica.

las mezclas, la durabilidad se reduce. Se pueden obtener valores aceptables de durabilidad con porcentajes de carbón menores al 9\%.

Sistema triangular de coordenadas para la resistencia a la compresión: Todas las briquetas superaron ampliamente la prueba de resistencia al a compresión, pero al analizar la Figura 5 se puede afirmar que los valores más altos de resistencia a la compresión se alcanzan cuando se manejan porcentajes de fibra de coco entre $60-70 \%$, cascarilla de arroz en un rango de $12-20 \%$ y carbón en proporciones inferiores al 10\%, aproximadamente.

Aglomerante de yuca: Observando con detalle las Figuras 4 y 5 , es notorio que el valor del aglomerante en cada uno de estos se encuentra fijo en $11,1 \%$, esto se debe a que, de todos los 4 factores presentes en las mezclas, el aglomerante es el que menos efectos tiene en cada una de las variables de respuesta.

Se fijó el porcentaje de aglomerante en 11,1\%, ya que este valor es el promedio del aglomerante total usado en cada una de las 40 corridas.

Relación entre durabilidad y porcentaje de carbón: De las 11 briquetas que tuvieron una durabilidad mayor al 90\%, la mayoría de estas tenían porcentajes de carbón menor al 10\%, es decir que mediante la Figura 6 se muestra una vez más que existe una correlación inversa negativa entre estas dos, ya que, 
Fuente: Design Expert versión 10.

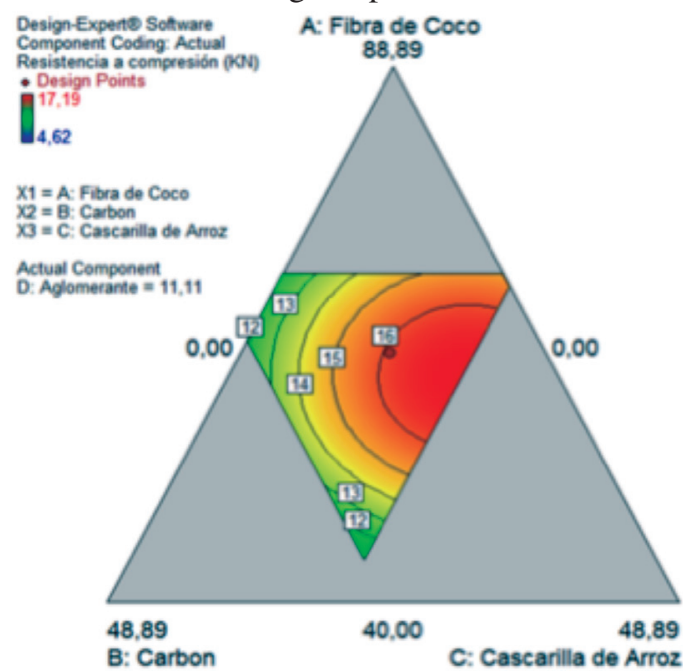

Figura 5. Gráfico de contorno para la resistencia a la compresión.

a menor porcentaje de carbón en las mezclas, mayor será la durabilidad mecánica de las briquetas.

Relación entre durabilidad y la fibra de coco: Un resultado importante se presenta a partir de la Figura 7, ya que en ella se muestra que todas las briquetas que poseen durabilidad mayor al $90 \%$, presentan porcentajes de fibra de coco superior al $63 \%$. Además, se muestra que en muchas otras

Fuente: Design Expert versión 10.

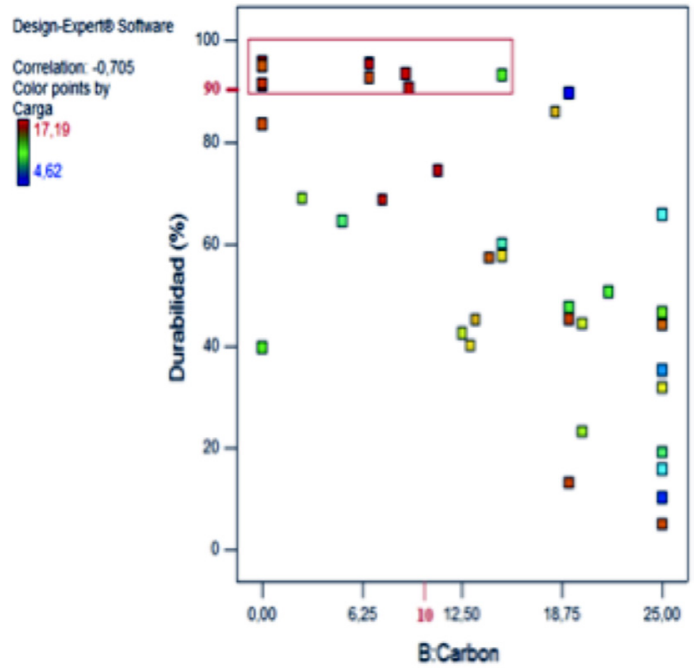

Figura 6. Durabilidad mecánica vs contenido de carbón.
Fuente: Design Expert versión 10.

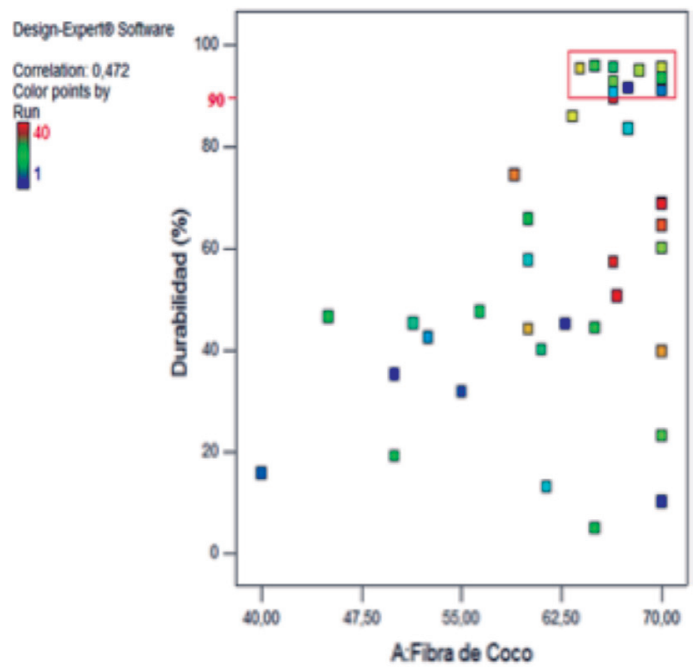

Figura 7. Durabilidad mecánica vs contenido de fibra de coco.

mezclas igualmente hay altos porcentajes de fibra de coco, pero con durabilidad menor al $90 \%$, esto quiere decir que es necesaria pero no suficiente la presencia de altos porcentajes de esta biomasa para conseguir buena durabilidad mecánica de las briquetas hechas con la mezcla biomasa-carbón-aglomerante.

Relación entre durabilidad y la cascarilla de arroz: A continuación, en la Figura 8, se muestra

Fuente: Design Expert versión 10.

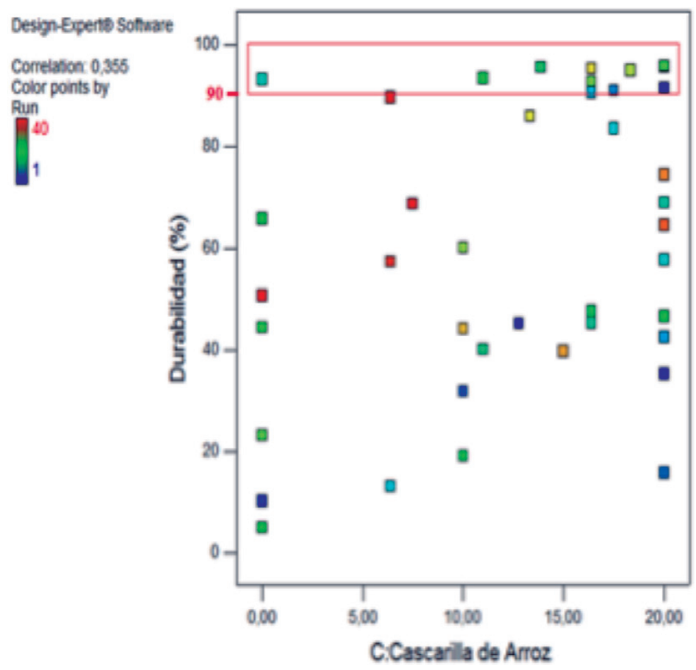

Figura 8. Durabilidad mecánica vs contenido de cascarilla de arroz. 
que las briquetas que alcanzaron una durabilidad mayor al $90 \%$ lo consiguieron con porcentajes de cascarilla de arroz muy variables entre mezclas, con rangos comprendidos entre 0-20\% de esta biomasa, esto quiere decir que no hay una correlación entre estas dos variables, pero analizando detalladamente el gráfico se puede afirmar que se puede conseguir buena durabilidad con la máxima cantidad de arroz usada en este experimento.

Mezclas óptimas: En la Tabla 5, se muestran las tres mezclas posibles, arrojadas por el software, luego de haber realizado la optimización multivariable.

Según los criterios establecidos, se obtuvieron tres soluciones, de las cuales el software recomienda seleccionar la número 1, sin embargo, es posible elegir cualquiera de las tres. Cabe resaltar, que todas las soluciones tienen valores en sus propiedades mecánicas dentro de los considerados aceptables para briquetas. En cuanto al poder calorífico, la mezcla número 3 es la que presenta un valor mayor, aunque no es significativa la diferencia de este valor con respecto a las otras dos soluciones.

Se observa que el porcentaje de carbón que recomienda el software es muy similar en las tres respuestas y las emisiones de azufre igualmente tienen valores similares y menores al $1 \%$ como lo reglamenta la resolución 623 , lo cual las convierte en una alternativa energética amigable con el medio ambiente.

Se puede afirmar que es posible darle uso a estas dos biomasas y al carbón mineral, para la elaboración de biocombustibles solidos densificados, contribuyendo así a la utilización de estas dos biomasas, las cuales son una gran fuente de energía pero que actualmente no tienen ningún valor adicional para los productores; además de darle provecho al carbón de la región en esta mezcla, consiguiendo de esta manera briquetas con $700 \%$ menos en las emisiones de azufre en comparación con la de este mineral en estado puro. Finalmente, es posible aumentar el poder calorífico de las briquetas en comparación con el de la cascarilla de arroz, sin embargo, este aumento no es significativo.

Para la prueba de laboratorio se seleccionó la muestra número 1, los resultados se observan en la Tabla 6:

Tabla 6. Resultados pruebas de laboratorio y error relativo.

\begin{tabular}{|l|c|c|c|}
\hline \multicolumn{1}{|c|}{ Propiedad } & $\begin{array}{c}\text { Valor } \\
\text { real } \\
\text { (Vr) }\end{array}$ & $\begin{array}{c}\text { Valor } \\
\text { software } \\
\text { (Vm) }\end{array}$ & $\begin{array}{c}\text { Error } \\
\text { relativo } \\
(\%)\end{array}$ \\
\hline $\begin{array}{l}\text { Poder calorífico } \\
(\mathrm{Mj} / \mathrm{Kg})\end{array}$ & 17,045 & 16,1 & 5,5 \\
\hline $\begin{array}{l}\text { Emisiones de } \\
\text { azufre (\%) }\end{array}$ & 0,2 & 0,214 & 7 \\
\hline
\end{tabular}

Fuente: Design Expert versión 10.

Como se puede observar en la Tabla 6, el error relativo existente para cada una de estas dos variables es relativamente bajo, lo cual indica que es posible calcular analíticamente el poder calorífico y las emisiones de azufre en función de las fracciones másicas de cada factor, tal y como lo recomendó la bibliografía, obteniéndose resultados muy acertados.

\section{CONCLUSIONES}

Se logró elaborar biocombustibles sólidos densificados (briquetas) a partir de la mezcla de biomasas residuales agroindustriales, carbón mineral y aglomerante de yuca.

Se evidencio que el carbón es el factor que más disminuye la durabilidad mecánica y la resistencia a la compresión de las briquetas.

Con la presión de compactación utilizada en esta investigación, se consiguió elaborar briquetas con

Tabla 5. Mezclas favorables según la optimización.

\begin{tabular}{|c|c|c|c|c|c|c|c|c|c|}
\hline Número & $\begin{array}{c}\text { Fibra } \\
\text { de coco }\end{array}$ & Carbón & Cascarilla & Aglom. & Carga & Durabilidad & $\begin{array}{c}\text { Poder } \\
\text { calorífico }\end{array}$ & Emisiones & Deseabilidad \\
\hline 1 & 61,2 & 7,7 & 20 & 11 & 17,1 & 100 & 16,1 & 0,214 & 0,671 \\
\hline 2 & 67,3 & 6,9 & 16,6 & 9,1 & 11,3 & 97 & 16,2 & 0,205 & 0,642 \\
\hline 3 & 70 & 7,1 & 14,4 & 8,3 & 10 & 93,6 & 16,3 & 0,21 & 0,589 \\
\hline
\end{tabular}

Fuente: Autores (2016). Design Expert versión 10. 
una relación altura/diámetro menor a 2, con lo cual se consigue que la geometría final de la briqueta le otorgue alta resistencia a la compresión.

Fue posible elaborar briquetas con durabilidad y resistencia a la compresión aceptables con el máximo porcentaje de cascarilla de arroz usada.

La fibra de coco es una biomasa que le otorga excelentes propiedades mecánicas a las briquetas cuando es usado en altos porcentajes de masa en la mezcla.

El factor que menos efecto tuvo sobre las variables de respuesta fue el aglomerante de yuca, dado que su poder calorífico es menor que el de las biomasas y el carbón y su contenido de azufre es despreciable. Además, su rango de trabajo se mantuvo en menor proporción.

Se consiguieron briquetas con propiedades mecánicas aceptables y con emisiones de azufre menor al $1 \%$, con porcentajes de carbón inferior al $10 \%$.

\section{REFERENCIAS}

[1] A. Bamgboye and S. Boluwafi. "Physical characteristics of briquettes from guinea corn (sorghum bi-color) residue". Department of Agricultural and Environmental Engineering. 2009.

[2] DANE. "Encuesta nacional de arroz mecanizado". 2011.

[3] K. Demirbas and A. Sahin. "Compacting of biomass for energy densification". Energy Sources, Part A: Recovery, Utilization, and Environmental Effects. pp. 1063-1068. 2009.

[4] E. Fonseca y L. Tierra. "Desarrollo de un proceso tecnológico para la obtención de briquetas de aserrín de madera y cascarilla de arroz, y pruebas de producción de gas pobre". Riobamba, Ecuador. 2011.

[5] O. Francis. "Evaluation of the effect of palm oil mill sludge on the properties of sawdust briquette". Renewable and Sustainable Energy Reviews. pp. 1749-1758. 2015.

[6] Gobernación de Córdoba. "Actividad carborifera en el municipio de puerto libertador”. Montería-Córdoba. 2010.

[7] P. Grover and M. Mishra. "Biomass Briquetting: Technology and Practice". Food and Agricultural Organisation of the United Nations, pp. 1-10. 1996.

[8] K. Ishii and T. Furuichi. "Influence of moisture content, particle size and forming temperature". Waste Management. pp. 2621-2626. 2014.

[9] N. Kaliyan and V. Morey. "Strategies to improve durability of switchgrass briquettes". American Society of Agricultural and Biological Engineers. pp. 1943-1953. 2009.

[10] P. Križan, L Šooš and D. Vukelič. "A study of impact technological parametres on the briquetting process". Working and Living Environmental Protection. pp. 39-47. 2009.

[11] S. Lee, B. Ahn, J. Choi, D.H. Han, G.S. Jeong, H.S. Ahn, S.H. and Yang I. "Effects of densification variables on the durability of wood pellets fabricated with Larix kaempferi C. and Liriodendron tulipifera L. sawdust". Biomass and Bioenergy. pp. 1-9. 2013.

[12] Y. Li and H. Liu. "High-pressure densification of wood residues to form an upgraded fuel". Biomass Bioenergy. Vol 19 N $^{\circ} 3$, pp. 177-186.

[13] C. Liu and S. Wu. "From biomass waste to biofuels and biomaterial building blocks". Renewable Energy. pp. 1-7. 2015.

[14] MADR. "Desarrollo de la fruticultura en Córdoba”. Montería. 2006.

[15] A. Miranda y R. Oliver. "La combustión". Barcelona: CEAC. 1996.

[16] A. Molina y G. Molina. "Manual de eficiencia energética térmica en la industria". Bilbao: CADEM. 1984.

[17] A. Moreno, R. Font and J. Conesa. "Physical and chemical evaluation of furniture waste briquettes". Waste Management. pp. 245252. 2016.

[18] R. Muazu and J. Stegemann. "Effects of operating variables on durability of fuel briquettes from rice". Fuel Processing Technology. pp. 137-145. 2015.

[19] J. Oladeji and C. Enweremadu. "The effects of some processing parameters on physical and densification characteristics of corncob briquettes". International Journal of Energy Engineering. Vol. $2 \mathrm{~N}^{\mathrm{o}}$ 1, pp. 22-27.

[20] J. Ortega y C. Berástegui. "Elaboración de biocombustibles sólidos densificados a partir de tres biomasas residuales, tres tipos de bioaglomerante y carbón mineral del Departamento de Córdoba". Montería Córdoba: Universidad de Córdoba. 2015. 
[21] M. Satin. "Functional Properties of Starches". Agricultural and Food Engineering Technologies Service (FAO). 1998.

[22] W Stelte, J. Holm and K. Sanadi. "A study of bonding and failure mechanisms in fuel pellets from different biomass resources". Biomass and Bioenergy. pp. 910-918. 2011.
[23] M. Stolarski, S. Szczukowski, J. Tworkowski, M. Krzyżaniak, P. Gulczyński and M. Mleczek. "Comparison of quality and production cost of briquettes made from agricultural and forest origin biomass". Renewable Energy. Vol. 57, pp. 20-26. 2013. 